\title{
MODULI OF MARKED RIEMANN SURFACES
}

\author{
BY BERNARD MASKIT ${ }^{1}$
}

Communicated by F. W. Gehring, August 2, 1973

The purpose of this note is to exhibit a set of complex analytic moduli for the space of closed Riemann surfaces of genus $g \geqq 2$, marked by a basis for the fundamental group. That one could find such moduli (i.e., biholomorphically embed the Teichmüller space of genus $g$ in $C^{3 g-3}$ ) was proven by Bers [2]. His moduli are variational-they depend on a choice of base surface. Our moduli are in some sense intrinsic, similar to the (real) moduli of Fenchel-Nielsen [4] and Keen [5]. In fact our moduli should be regarded as the complex analogue of the Fenchel-Nielsen and Keen moduli. (The geometric relationship between these different moduli is clear, and they are real-analytically equivalent.)

The actual expressions for the moduli given below involve multiplicative constants and square roots. These normalizations serve two purposes. First, the moduli space is contained in a product of half-planes. Second, with these normalizations, the group of translations

$$
z \rightarrow z+n, \quad n=\left(n_{1}, \cdots, n_{3 g-3}\right) \in Z^{3 g-3},
$$

is a subgroup of the modular group; i.e., two points of the space of moduli which are identified under (1) correspond to the same Riemann surface with different markings.

The moduli occur as moduli of a set of generators of a Kleinian group; these are defined by traces of loxodromic elements and cross-ratios of fixed points of parabolic elements. Each of the $3 g-3$ coordinates is determined by a subgroup of the Kleinian group; using this one sees that each of the coordinates can be identified, in a natural way, with the modulus of a torus.

In this note we present proofs only in very broad outline-details will appear elsewhere.

1. Let $S$ be a closed Riemann surface of genus $g \geqq 2$, and let $A_{1}, B_{1}$, $\cdots, A_{g}, B_{g}$ be a canonical homotopy basis on $S$ (we regard $A_{1}, \cdots$, $B_{g}$ as being both a set of loops on $S$ and as a set of generators for $\pi_{1}(S)$ ).

AMS (MOS) subject classifications (1970). Primary 30A46.

${ }^{1}$ Research supported by NSF grant PO19572000. 
We pick out the following set of $3 g-3$ elements of $\pi_{1}(S)$ :

$$
\begin{aligned}
& B_{i}, i=1, \cdots, g ; \\
& C_{i}=\left[B_{i}, A_{i}\right]=B_{i} A_{i} B_{i}^{-1} A_{i}^{-1}, i=1, \cdots, g ; \\
& D_{i}=\prod_{j=1}^{i+1}\left[B_{j}, A_{j}\right]=B_{i+1} \cdots A_{1}^{-1}, i=1, \cdots, g-3 .
\end{aligned}
$$

For $g=2, C_{1}=C_{2}^{-1}$ and our set consists of $B_{1}, B_{2}$ and $C_{1}$.

If we do not require our loops to pass through a common base point, then the above elements are a maximal set of $3 g-3$ homotopically distinct simple disjoint loops on $S$. These loops are shown in Figure 1 for the case $g=5$.

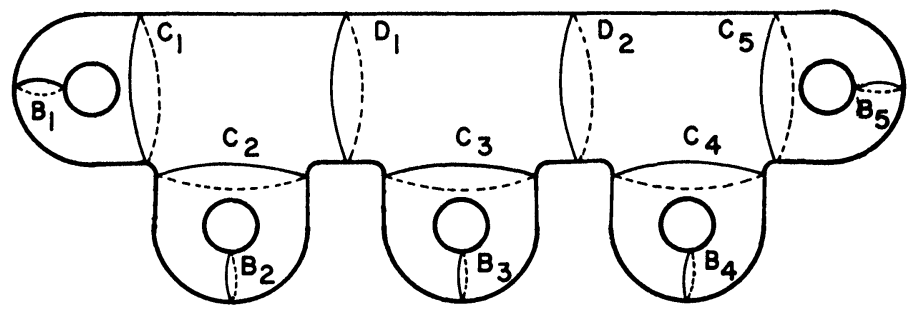

FIGURE 1

We remark that our particular moduli depend on this particular choice of $3 g-3$ homotopically distinct simple disjoint loops. We could have chosen a different set of $3 g-3$ loops and obtained a different set of moduli with the same properties.

We want to describe an isomorphism from $\pi_{1}(S)$ onto a Kleinian group without reference to variations from a given group. For fixed $g \geqq 2$, we set $K_{g}=(\operatorname{PSL}(2 ; C))^{2 g}$ factored by the relations

$$
\left(x_{1}, \cdots, x_{2 g}\right) \sim\left(y x_{1} y^{-1}, \cdots, y x_{2 g} y^{-1}\right), \quad y \in \operatorname{PSL}(2 ; C) .
$$

THEOREM 1. Let $S$ be a Riemann surface of genus $g \geqq 2$, with a canonical homotopy basis $A_{1}, \cdots, B_{g}$. There is a unique point $\tau \in K_{g}$, represented as $\left(a_{1}, b_{1}, \cdots, a_{g}, b_{g}\right)$ satisfying the following properties.

(i) The group $G$ generated by $a_{1}, \cdots, b_{g}$ is Kleinian and has a simply connected invariant component $\Delta$ of its region of discontinuity.

(ii) $G$ acts freely on $\Delta$, and $\Delta / G=S$.

(iii) There are base points $o \in S$ and $\tilde{o} \in \Delta$, lying over $o$, so that the natural isomorphism $\pi_{1}(S, o) \rightarrow G$ maps $A_{i} \rightarrow a_{i}, B_{i} \rightarrow b_{i}, i=1, \cdots, g$.

(iv) The following elements of $G$ are parabolic:

$$
\begin{aligned}
b_{i}, & i=1, \cdots, g, \\
c_{i} & =\left[b_{i}, a_{i}\right]=b_{i} a_{i} b_{i}^{-1} a_{i}^{-1}, \quad i=1, \cdots, g, \\
d_{i} & =\sum_{j=1}^{i+1}\left[b_{j}, a_{j}\right] .
\end{aligned}
$$


The proof of Theorem 1 involves a geometric construction [7], Bers' technique of variation of parameters using quasiconformal mappings [3], and Marden's isomorphism theorem [6].

We remark that the elements $b_{i}, c_{i}$ and $d_{i}$ correspond to the loops $B_{i}$, $C_{i}$ and $D_{i}$ shown in Figure 1.

Theorem 1 can be regarded as giving a bijective mapping $\Phi$ from the Teichmüller space of genus $g$, starting with some base surface, onto $K_{g}$. This map $\Phi$ is actually defined in terms of quasiconformal mappings, and so $\Phi$ is holomorphic (see Ahlfors-Bers [1]).

2. We next describe parameters for $K_{g}$. Using Theorem 1, each point $\tau \in K_{g}$ defines a Kleinian group $G_{r}$, up to conjugation. If $g$ is an element of $G_{\tau}$, then $\operatorname{tr}(g)$, the trace of $g$ is a well-defined function on $K_{g}$, up to multiplication by -1 . Similarly if $g_{1}, g_{2}, g_{3}, g_{4}$ are parabolic elements of $G_{\tau}$, with distinct fixed points $x_{1}, \cdots, x_{4}$, respectively, then

is a well-defined function on $K_{g}$.

$$
\operatorname{cr}\left(g_{1}, g_{2} ; g_{3}, g_{4}\right)=\frac{x_{1}-x_{4}}{x_{1}-x_{2}} \cdot \frac{x_{3}-x_{2}}{x_{3}-x_{4}}
$$

Theorem 2 (General CASE). Let $g \geqq 5$. The functions

$$
\begin{aligned}
\beta_{i} & =(i / 2) \operatorname{tr} a_{i}, \quad i=1, \cdots, g, \\
\gamma_{1} & =\frac{1}{2} \operatorname{cr}^{1 / 2}\left(b_{1}, a_{1} b_{1} a_{1}^{-1} ; d_{1}, c_{2}\right), \\
\gamma_{2} & =\frac{1}{2} \operatorname{cr}^{1 / 2}\left(b_{2}, a_{2} b_{2} a_{2}^{-1} ; c_{1}, d_{1}\right), \\
\gamma_{i} & =\frac{1}{2} \operatorname{cr}^{1 / 2}\left(b_{i}, a_{i} a_{i}^{-1} ; d_{i-2}, d_{i-1}\right), \quad i=3, \cdots, g-2, \\
\gamma_{g-1} & =\frac{1}{2} \operatorname{cr}^{1 / 2}\left(b_{g-1}, a_{g-1} b_{g-1} a_{g-1}^{-1} ; d_{g-3}, c_{g}\right), \\
\gamma_{g} & =\frac{1}{2} \operatorname{cr}^{1 / 2}\left(b_{g}, a_{g} b_{g} a_{g}^{-1} ; c_{g-1}, d_{g-3}\right), \\
\delta_{1} & =\frac{1}{2} \operatorname{cr}^{1 / 2}\left(c_{2}, c_{1} ; d_{2}, c_{3}\right), \\
\delta_{i} & =\frac{1}{2} \operatorname{cr}^{1 / 2}\left(c_{i+1}, d_{i-1} ; d_{i+1}, c_{i+2}\right), \quad i=2, \cdots, g-4, \\
\delta_{g-3} & =\frac{1}{2} \operatorname{cr}^{1 / 2}\left(c_{g-2}, d_{g-4} ; c_{g}, c_{g-1}\right)
\end{aligned}
$$

can all be chosen to have positive imaginary part in $K_{g}$. With this choice, these $(3 g-3)$ functions yield a biholomorphic embedding of $K_{g}$ as an open set in $C^{3 g-3}$.

There are versions of Theorem 2 for genera 2, 3 and 4 . The statement of the theorem is the same, only the parameters are different. We simply list below the correct parameters for these lower genera.

In all cases we have $\beta_{i}, i=1, \cdots, g$, as above. 
In genus 2 , there is just

$$
\gamma_{1}=\frac{1}{2} \operatorname{cr}^{1 / 2}\left(b_{1}, a_{1} b_{1} a_{1}^{-1}, b_{2}, a_{2} b_{2} a_{2}^{-1}\right) .
$$

In genus 3 , we have

$$
\begin{aligned}
& \gamma_{1}=\frac{1}{2} \operatorname{cr}^{1 / 2}\left(b_{1}, a_{1} b_{1} a_{1}^{-1} ; c_{3}, c_{2}\right), \\
& \gamma_{2}=\frac{1}{2} \operatorname{cr}^{1 / 2}\left(b_{2}, a_{2} b_{2} a_{2}^{-1} ; c_{1}, c_{3}\right), \\
& \gamma_{3}=\frac{1}{2} \operatorname{cr}^{1 / 2}\left(b_{3}, a_{3} b_{3} a_{3}^{-1} ; c_{2}, c_{1}\right) .
\end{aligned}
$$

In genus 4 , the $\gamma_{i}$ are as in the general case and

$$
\delta_{1}=\frac{1}{2} \operatorname{cr}^{1 / 2}\left(c_{2}, c_{1} ; c_{4}, c_{3}\right) \text {. }
$$

3. Each of the parameters $\beta_{i}$ corresponds naturally to $B_{i}, \gamma_{i}$ to $C_{i}$, and $\delta_{i}$ to $D_{i}$.

The cross-ratios used to obtain the $\gamma_{i}$ and $\delta_{i}$ are obtained geometrically as follows. In Figure $1, C_{i}$ is "adjacent" to $B_{i}, D_{i-2}$ and $D_{i-1}$. If we cut the surface along these curves we get a sphere with four holes-these holes correspond to the four elements that appear in the cross-ratio defining $\gamma_{i}$. The cross-ratios appearing in $\delta_{i}$ are obtained in the same manner.

The four elements $b_{i}, a_{i} b_{i} a_{i}^{-1}, d_{i-2}$ and $d_{i-1}$ appearing in the definition of $\gamma_{i}$ generate a subgroup $H$ of $G_{\tau}$. This subgroup has a simply-connected invariant component $\Delta(H)$ of its set of discontinuity. One easily sees that $H$ is uniquely determined, up to conjugation, by $\gamma_{i}$, and that $\Delta(H) / H$ is a 4-times punctured sphere. Of course $H$ comes with a set of generators, so $\gamma_{i}$ locates a point in the Teichmüller space of 4-times punctured spheres. There is, of course, a canonical bijection between this Teichmüller space and the Teichmüller space of tori. We can holomorphically identify $\gamma_{i}$ with the modulus of a torus ( $\gamma_{i}$ has positive imaginary part, but this identification is almost undoubtedly not the identity).

Exactly as above, each $\delta_{i}$ can be identified with the modulus of a torus. The parameters $\beta_{i}$ are more direct. The subgroup $H$ of $G$ generated by $a_{i}$ and $b_{i}$ has a simply connected invariant component $\Delta(H)$; here $\Delta(H) / H$ is a punctured torus. Since every torus is homogeneous, we can holomorphically identify $\beta_{i}$ with the modulus of a torus (again this identification is almost undoubtedly not the identity).

4. For each of the loops $B_{i}, C_{i}$ and $D_{i}$, there is a cyclic subgroup of the modular group generated by the Dehn twist or screw map about that loop. These maps can be realized in a very simple form in our parameter space. The twist about $B_{i}$ corresponds to the translation $\beta_{i} \rightarrow \beta_{i}+1$; the twist about $C_{i}$ corresponds to the translation $\gamma_{i} \rightarrow \gamma_{i}+1$; and the twist about $D_{i}$ corresponds to the translation $\delta_{i} \rightarrow \delta_{i}+1$. 
To sum up, we have a biholomorphic embedding of the Teichmüller space of genus $g$ in $C^{3 g-3}$; the image is contained in a product of halfplanes-it also contains a product of half-planes; the image is invariant under the translation group (1) and this group of translations is a subgroup of the modular group.

\section{REFERENCES}

1. L. Ahlfors and L. Bers, Riemann's mapping theorem for variable metrics, Ann. of Math. (2) 72 (1960), 385-404. MR 22 \#5813.

2. L. Bers, On moduli of Riemann surfaces (mimeographed lecture notes), Eidgenossische Technische Hochschule, Zurich, 1964.

3. - Uniformization by Beltrami equations, Comm. Pure Appl. Math. 14 (1961), 215-228. MR 24 \#A2022.

4. W. Fenchel and J. Nielsen, Discontinuous groups of non-Euclidean motions (unpublished manuscript).

5. L. Keen, Intrinsic moduli on Riemann surfaces, Ann. of Math. (2) 84 (1966), 404420. MR 34 \#2859.

6. A. Marden, The geometry of finitely generated Kleinian groups, Ann. of Math. (to appear).

7. B. Maskit, On boundaries of Teichmüller spaces and on Kleinian groups. II, Ann. of Math. (2) 91 (1970), 607-639. MR 45 \#7045.

Department of Mathematics, State University of New York at Stony Brook, StONY BroOK, New YorK 11790 